\title{
UNE CARTE CONCEPTUELLE POUR LE PROGRAMME DE BACCALAURÉAT EN GÉNIE CHIMIQUE À POLYTECHNIQUE MONTRÉAL
}

\author{
Édith Sauvageau-Grenier, Jason R. Tavares, Nick Virgilio et Patrice Farand \\ edith.sauvageau-grenier@polymtl.ca
}

Il est important pour un étudiant en génie chimique de bien comprendre les concepts généraux associés à sa formation afin de maintenir et d'accroitre sa motivation au cours de ses études. Pour atteindre cet objectif, des interactions et des liens entre les différents cours doivent cependant exister et être clairement identifiés. Un niveau d'interaction approprié entre les cours permet à l'étudiant d'établir des liens entre ses apprentissages et d'apprécier la pertinence de ses cours. À Polytechnique Montréal, le département de génie chimique veut évaluer, améliorer et optimiser le niveau d'interaction qui devrait exister entre les différents cours du programme à l'aide d'une carte conceptuelle. Cette carte conceptuelle présente des idées structurées hiérarchiquement, avec des liens clairs et faciles à comprendre. Elle tente d'organiser les idées de façon similaire à notre cerveau. C'est un outil qui, avec sa présentation visuelle facile à comprendre, permet d'optimiser les liens entre les cours d'un curriculum tout en évitant les redondances. Elle peut également être utilisée à des fins de formation et pour l'évaluation des apprentissages chez les étudiants. Il pourrait donc être intéressant d'intégrer cet outil dans les cours afin d'évaluer la compréhension des étudiants.

La carte conceptuelle réalisée dans le cadre du présent projet est constituée de deux niveaux de concepts. Le premier niveau représente les quatre piliers principaux d'un programme de génie chimique (Figure 1): les sciences fondamentales, les sciences du génie, les notions complémentaires et la conception. Le deuxième niveau (Figure 2) représente les sous-concepts associés aux concepts du premier niveau. Ce deuxième niveau comprend les différents types de liens perçus entre ces sous-concepts. Ces liens ont été illustrés de différentes manières pour différencier, par exemple, les liens perçus intuitivement (flèches pleines) de ceux perçus suite à la lecture des différentes descriptions de cours de diverses universités et à des discussions par rapport aux premières ébauches de la carte (flèches pointillées). La réalisation de cette carte et l'évolution des liens établis a permis de constater les lacunes, du point de vue d'une étudiante, du programme de génie chimique. Des analyses et recommandations ont pu en être retirées; les principales étant d'effectuer des rappels de notions d'un cours à l'autre, d'optimiser la présentation non-linéaire de la matière dans les cours puis de renforcer la théorie vue en cours à l'application concrète sur le marché du travail en ce qui concerne l'ingénierie des systèmes. Il serait intéressant d'avoir la perception de plusieurs étudiants finissants afin d'avoir un échantillon global d'analyse du programme plus représentatif.

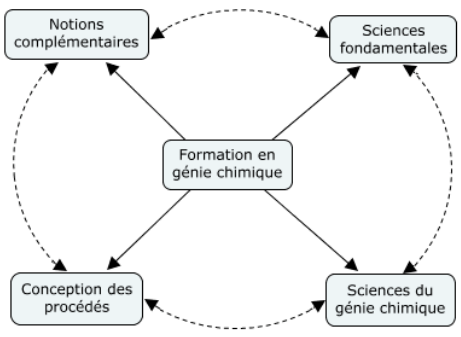

Figure 1 : Premier niveau de concepts

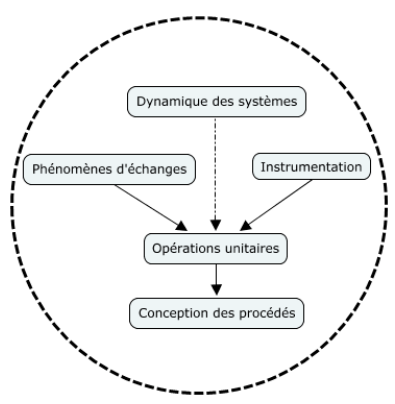

Figure 2 : Exemple d'un deuxième niveau de concepts illustrant les liens autour du cours d'opérations unitaires offert à la $3^{\mathrm{e}}$ année 